Article

\title{
Large Uniaxial Magnetic Anisotropy of Hexagonal Fe-Hf-Sb Alloys
}

\author{
Lukas Kyvala $^{1,2}{ }^{\oplus}$, Maxim Tchaplianka ${ }^{1}$, Alexander B. Shick ${ }^{1, *}$ and Sergii Khmelevskyi ${ }^{3}$ \\ and Dominik Legut ${ }^{2}$ (D) \\ 1 Institute of Physics, Czech Academy of Sciences, Na Slovance 2, CZ-18221 Prague, Czech Republic; \\ lukas.kyvala@vsb.cz (L.K.); tchaplianka@fzu.cz (M.T.) \\ 2 IT4Innovations and Nanotechnology Centre, VSB-Technical University of Ostrava, 17. listopadu 2172/15, \\ CZ-70800 Ostrava-Poruba, Czech Republic; dominik.legut@vsb.cz \\ 3 Center for Computational Materials Science, Vienna University of Technology, Wiedner Hauptstrasse 8-10, \\ A-1040 Vienna, Austria; sk@iap.tuwien.ac.at \\ * Correspondence: shick@fzu.cz
}

Received: 4 May 2020; Accepted: 25 May 2020; Published: 27 May 2020

check for updates

\begin{abstract}
We theoretically investigate the electronic and magnetic structure of $\mathrm{Fe}_{2} \mathrm{Hf}$. The density functional theory calculations are shown to produce the negative, easy-plane, magnetic anisotropy in the hexagonal $\mathrm{Fe}_{2} \mathrm{Hf}$. Antimony substitution suppresses the planar magnetization direction and favors the uniaxial magnetic anisotropy, in agreement with experimental observations. Our study suggests the possibility of the chemical control of the magnetic anisotropy in $\mathrm{Fe}_{2} \mathrm{Hf}$ by $\mathrm{Sb}$ substitution, and illustrates the potential of $(\mathrm{Fe}, \mathrm{Sb})_{2+x} \mathrm{Hf}_{1-x}$ Laves phase alloys for the permanent magnet applications.
\end{abstract}

Keywords: electronic structure; magnetism; magnetic anisotropy; permanent magnets

\section{Introduction}

Permanent magnets are an indispensable part of the modern technology [1]. They are mainly used for data storage and energy conversion, and are present in smartphones; laptops; audio and video devices; and industrial applications, such as electrical motors and wind turbines. There is a high demand for permanent magnets (PMs) with high performance for efficient renewable energy production and conversion [2]. Research targeting new PMs is driven by both technological and economical factors, to keep costs low and ensure supply stability.

A good PM has to have a high Curie temperature, large magnetization density, and coercivity. The large coercivity is required to resist demagnetization, and advanced PM should achieve a coercivity $\mu_{0} H_{c}>1.5 \mathrm{~T}$ [3]. It is determined by the magneto-crystalline anisotropy energy (MAE), the energy to rotate the magnetization from the easy to the hard direction. This large coercivity can be provided by uniaxial magnetic phases with hexagonal, rhombohedral, or tetragonal crystal structures with the high $\mathrm{MAE}>1 \mathrm{MJ} / \mathrm{m}^{3}$.

The best known PMs are based on $\mathrm{Nd}_{2} \mathrm{Fe}_{14} \mathrm{~B}$ with the most superior PM properties [4], and $\mathrm{SmCO}_{5}$ [5] which is used for the high temperature PM applications. These materials belong to the class of the rare-earth (RE)-transitional metal $(3 d)$ intermetallics. In these materials, the RE element provides high value of the MAE, and the $3 d$ metal component yields a high magnetization density and a high Curie temperature.

In recent years, major effort has been made to search for the RE-free PM materials [6]. The reason is a growing concern for the RE elements' (Nd, Sm, Dy) sustainability, together with growing demand from the industry for cheap and stable high-temperature PMs [2]. So far, the research 
has followed two routes: (i) to search directly for the transition metal based intermetallics in hexagonal, rhombohedral, or tetragonal crystal structures with the high MAE; (ii) to find a modification of already known ferromagnets by substitutional and interstitial alloying to increase the MAE.

Great prospects in the second direction were demonstrated by Coey and Sun [7] who have observed strong MAE increase in the iron-based RE intermetalics with substitution of the interstitial nitrogen. Furthermore, recently it was proposed experimentally and theoretically that alloying $6.25 \%$ of $\mathrm{Sb}$ into the hexagonal ferromagnet $\mathrm{Fe}_{3} \mathrm{Sn}$ changes the MAE and turns the magnetization direction from the easy-plane to the easy axis [8]. Another successful example of the chemical engineering of the MAE is the recent experimental observation of a large uniaxial MAE in $\mathrm{Fe}_{2} \mathrm{Hf}$ with $\mathrm{Sb}$ substitution [9].

The Fe-based Laves phases with 1:2 stoichiometry were never considered as candidates for magnetically hard materials. They usually crystallize in the cubic C15 structure with the small MAE, and posses superior magnetostrictive properties (e.g., giant magnetostriction in rare-earth- $\mathrm{Fe}_{2}$ [10]). Experimentally for the stoichiometric $\mathrm{HfFe}_{2}$, the cubic $\mathrm{C} 15$ phase is slightly more stable than the hexagonal C14 phase at the low temperatures [11], and both phases were reported to co-exist for high temperatures [12].

It was shown experimentally [9] that the Sb substitution stabilizes Fe-Hf-Sb alloy in hexagonal $\mathrm{C} 14$ phase, and leads to the large positive MAE of $\sim 1.5 \mathrm{MJ} / \mathrm{m}^{3}$ for $(\mathrm{Fe}, \mathrm{Sb})_{2+x} \mathrm{Hf}_{1-x}$ alloy with $13.5 \%$ of $\mathrm{Sb}$. Together with saturation magnetic polarization of $\sim 0.8 \mathrm{MA} / \mathrm{m}(1 \mathrm{~T})$, and the Curie temperature of $470 \mathrm{~K}$, these properties make the alloy a promising candidate for the PM applications.

In this work, we aim to investigate the MAE in the hexagonal $\mathrm{Fe}_{2} \mathrm{Hf}$ compound, and the Fe-Hf-Sb alloy making use of the density functional theory (DFT) implemented in the projector augmented-wave VASP [13], the full-potential linearized augmented plane wave (FLAPW) [14], and the Korringa-Kohn-Rostokker (KKR) [15] methods.

We show theoretically that the $\mathrm{Sb}$ substitution in hexagonal $\mathrm{Fe}_{2} \mathrm{Hf}$ leads to the strong uniaxial MAE in good quantitative agreement with the experimental data. We demonstrate that the role of $\mathrm{Sb}$ substitution is not limited by stabilization of the $\mathrm{Fe}_{2} \mathrm{Hf}$ in hexagonal $\mathrm{C} 14$ structure. The large uniaxial MAE occurs due to the presence of the $\mathrm{Sb}$ atoms in the crystalline matrix. Apart from the electronic mechanism, the structure relaxation plays an essential role in the MAE increase. In addition, we evaluate the inter-atomic exchange interactions in $\mathrm{Fe}_{2} \mathrm{Hf}$, and make an estimate for the Curie temperature.

\section{Results}

\subsection{Hexagonal $\mathrm{Fe}_{2} \mathrm{Hf}$}

The $\mathrm{Fe}_{2} \mathrm{Hf}$ has hexagonal $\mathrm{C} 14$ crystal structure with $P 6_{3} / m m c$ (number 194) space group and contains twelve atoms per unit cell (see Figure 1A). There are four formula units in the unit cell. The Fe atoms occupy (2a) and (6h) Wyckoff positions, and Hf is in (4f) Wyckoff position (see Table 1). First, we performed the scalar-relativistic VASP, KKR, and FLAPW calculations without spin-orbit coupling (SOC). The experimental lattice constants $a=4.968$ and $c=8.098$, and internal positions [16] shown in Table 1 were used in the calculations. The FLAPW calculated magnetic moments inside "muffin-tin" (MT) spheres for different Fe and Hf atoms in the unit cell together with the total magnetic moment per cell are shown in Table 1. The VASP and KKR calculations yield very similar results. Note that the magnetic moments are aligned in the same direction for the both Fe-sublattices, and the Hf moments are pointed in the opposite direction.

The spin-projected total density of states (DOS), as a result of FLAPW calculations without spin-orbit coupling (SOC), is shown in Figure 2A, and the $d$-states projected DOS for Fe and Hf atoms in different Wyckoff positions are shown in Figure 2B. Since the magnetic moments of Fe and Hf are anti-aligned, the exchange splitting of Fe and Hf is opposite. Virtually all the DOS character near $E_{F}$ is from Fe $d$-states. Both Fe and Hf-projected DOS illustrate itinerant character of the $d$-states. The calculated total (spin-up plus spin-down) DOS( $\left.E_{F}\right)$ for $\mathrm{Fe}_{2} \mathrm{Hf}$ of $1.0751 / \mathrm{eV}$ per Fe atom is close 
to the corresponding DOS value of $1.111 / \mathrm{eV}$ for the $b c c$-Fe. Since the Fe-atom moments in $\mathrm{Fe}_{2} \mathrm{Hf}$ (see Table 1) are smaller than $2.2 \mu_{B}$ moment for $b c c-\mathrm{Fe}$, the moment formation in $\mathrm{Fe}_{2} \mathrm{Hf}$ is weaker.

Table 1. The calculated magnetic moments (in $\mu_{B}$ ) inside the MT-spheres of different atoms in pristine $\mathrm{Fe}_{2} \mathrm{Hf}$ and Fe58-Hf25-Sb17 alloy unit cell, together with the total magnetic moment per cell. The Wyckoff positions and internal coordinates of different atoms in the unit cell are shown.

\begin{tabular}{|c|c|c|c|c|c|}
\hline \multirow[b]{2}{*}{$\begin{array}{l}\text { Wyckoff } \\
\text { Positions }\end{array}$} & \multirow[b]{2}{*}{ Element } & \multicolumn{2}{|c|}{$\mathrm{Fe}_{2} \mathrm{Hf}$} & \multicolumn{2}{|c|}{ Fe58-Hf25-Sb17 } \\
\hline & & $\begin{array}{l}\text { Internal } \\
\text { Position }\end{array}$ & Magnetic Moments & Element & Magnetic Moments \\
\hline$(2 a)$ & $\mathrm{Fe}(1)$ & $(0,0,0)$ & 1.71 & $\mathrm{Sb}$ & -0.03 \\
\hline$(2 a)$ & $\mathrm{Fe}(2)$ & $(0,0,1 / 2)$ & 1.71 & $\mathrm{Fe}$ & 2.00 \\
\hline (6h) & $\mathrm{Fe}(3)$ & $(0.17,0.34,1 / 4)$ & 1.74 & $\mathrm{Fe}$ & 1.64 \\
\hline (6h) & $\mathrm{Fe}(4)$ & $(-0.34,-0.17,1 / 4)$ & 1.74 & $\mathrm{Fe}$ & 1.64 \\
\hline (6h) & $\mathrm{Fe}(5)$ & $(-0.17,-0.34,-1 / 4)$ & 1.74 & $\mathrm{Fe}$ & 1.55 \\
\hline (6h) & $\mathrm{Fe}(6)$ & $(0.34,0.17,-1 / 4)$ & 1.74 & $\mathrm{Fe}$ & 1.55 \\
\hline (6h) & $\mathrm{Fe}(7)$ & $(0.17,-0.17,1 / 4)$ & 1.74 & $\mathrm{Fe}$ & 1.64 \\
\hline (6h) & $\mathrm{Fe}(8)$ & $(-0.17,0.17,-1 / 4)$ & 1.74 & $\mathrm{Fe}$ & 1.55 \\
\hline$(4 \mathrm{f})$ & $\mathrm{Hf}(1)$ & $(1 / 3,2 / 3,0.563)$ & -0.35 & Hf & -0.28 \\
\hline (4f) & $\operatorname{Hf}(2)$ & $(2 / 3,1 / 3,0.063)$ & -0.35 & Hf & -0.30 \\
\hline (4f) & $\operatorname{Hf}(3)$ & $(1 / 3,2 / 3,-0.063)$ & -0.35 & Hf & -0.27 \\
\hline (4f) & Hf (4) & $(2 / 3,1 / 3,-0.563)$ & -0.35 & $\mathrm{Sb}$ & 0.01 \\
\hline \multicolumn{3}{|c|}{ Total magnetic moment } & 13.48 & & 10.48 \\
\hline
\end{tabular}
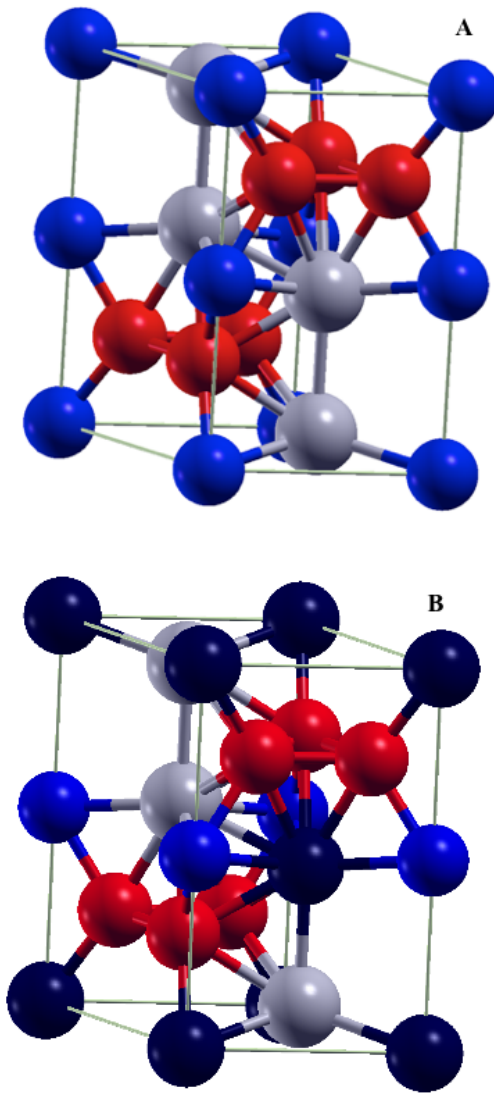

Figure 1. Crystal structure (hexagonal C14) for pristine $\mathrm{Fe}_{2} \mathrm{Hf}(\mathbf{A})$. Iron atoms in (2a) Wyckoff positions are represented by red-colored balls, iron atoms in (6h) Wyckoff positions are represented by blue-colored balls, and hafnium atoms in (4f) Wyckoff positions are represented by gray balls. (B) The unit cell of Fe58-Hf25-Sb17 alloy in the lowest energy configuration. Antimony atoms are shown by dark-blue-colored balls. 
A

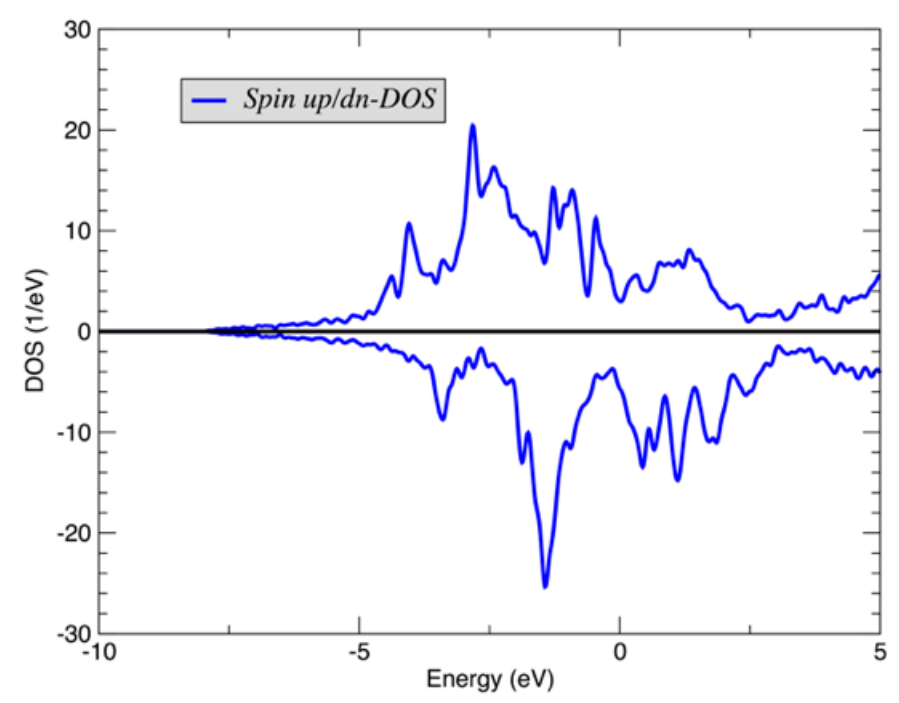

B

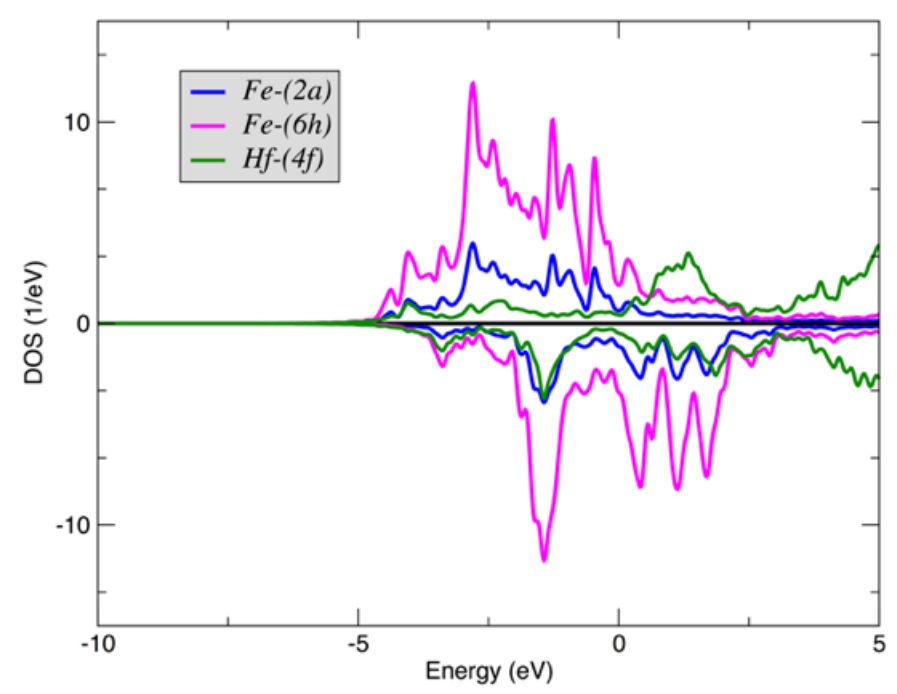

Figure 2. Density of states (DOS) of the $\mathrm{Fe}_{2} \mathrm{Hf}$ : (A) Spin-projected total DOS. (B) Spin-projected DOS for the $d$-states of Fe and Hf atoms in different Wyckoff positions. The Fermi level $E_{F}$ is set to $E_{F}=0$.

In order to estimate the Curie temperature we calculate the inter-site pair exchange interactions $J_{i j}$ of the classical Heisenberg Hamiltonian,

$$
\hat{H}=-\sum_{i, j} J_{i, j} \vec{e}_{i} \vec{e}_{j}
$$

where $\vec{e}_{i}$ and $\vec{e}_{j}$ mark the unit directional vectors of the magnetic moments at $i$ and $j$ different $\mathrm{Fe}$ lattice sites for $\mathrm{Fe}_{2} \mathrm{Hf}$. The calculations were performed making use of the magnetic force theorem [17] implemented in the bulk KKR formalism [18]. Since the magnetic ordering happens out of high temperature paramagnetic state, the exchange interactions were evaluated in the disordered local moment (DLM) [19] model, used to treat the thermal magnetic disorder. The importance of using the DLM reference state for estimation of the Curie temperature is demonstrated in [20]. 
The calculated exchange constants are shown in the Figure 3. There are three types of the inter-site exchange interactions: intra-sublattice interactions between Fe moments on (2a)-(2a) sites and (6h)-(6h) sites, and inter-sublattice (2a)-(6h) exchange coupling. It is seen from the Figure 3 that the exchange interactions have a long range, as expected for the metallic magnet. The (6h)-(6h) intra-sublattice exchange couplings are the strongest, and provide main contribution to the Curie temperature. The inter-sublattice exchange coupling between the (2a) and (6h) first nearest neighbors is also strong. Thus, it is expected that the magnetization of (2a) sublattice will have similar temperature behavior to the magnetization of (6h) sublattice. The mean-field theory estimate yields $445 \mathrm{~K}$ for the Curie temperature. This value is in fair agreement with the value of $470 \mathrm{~K}$ experimentally determined for Fe-Hf-Sb alloys [9].

The energy penalty of changing the orientation of the magnetization from in-plane to out-of-plane is called the magnetic anisotropy energy (MAE). Note that for a hexagonal crystal symmetry, the leading term in the MAE angular dependence is $K_{1} \sin ^{2} \theta$, with $\theta$ the angle between the magnetization and crystallographic $c$-axis. The MAE is then computed as the total energy difference when magnetization is oriented along [1100] and [0001] crystal axes, MAE $=E[1100]-E[0001]$.

Starting from the scalar-relativistic calculations, we perform the self-consistent total energy calculations with the SOC included, making use of a relativistic version of FLAPW [21]. The conventional local spin density approximation (von Barth-Hedin) is adopted, which we expect to be valid for itinerant metallic systems. In these calculations, the magnetization is fixed along [1100] and [0001] axes. The special k-points method is used for the Brillouin zone (BZ) integration with the Gaussian smearing of $1 \mathrm{mRy}$ for k-points weighting, and the equivalent to about 3200 k-points in the full BZ are used. Importantly, the same set of k-points must be used for different directions of the magnetization, in order to achieve accurate MAE results. The calculated MAE $=-0.57 \mathrm{meV}$ per unit cell $\left(-0.54 \mathrm{MJ} \mathrm{m}^{-3}\right)$ indicates the "in-plane" preferential direction of the magnetization in pristine $\mathrm{Fe}_{2} \mathrm{Hf}$. Although hexagonal $\mathrm{Fe}_{2} \mathrm{Hf}$ has a saturation magnetic moment and the Curie temperature desirable for a permanent magnet applications, the magnetization lies in the hexagonal plane, which is detrimental for PM material.

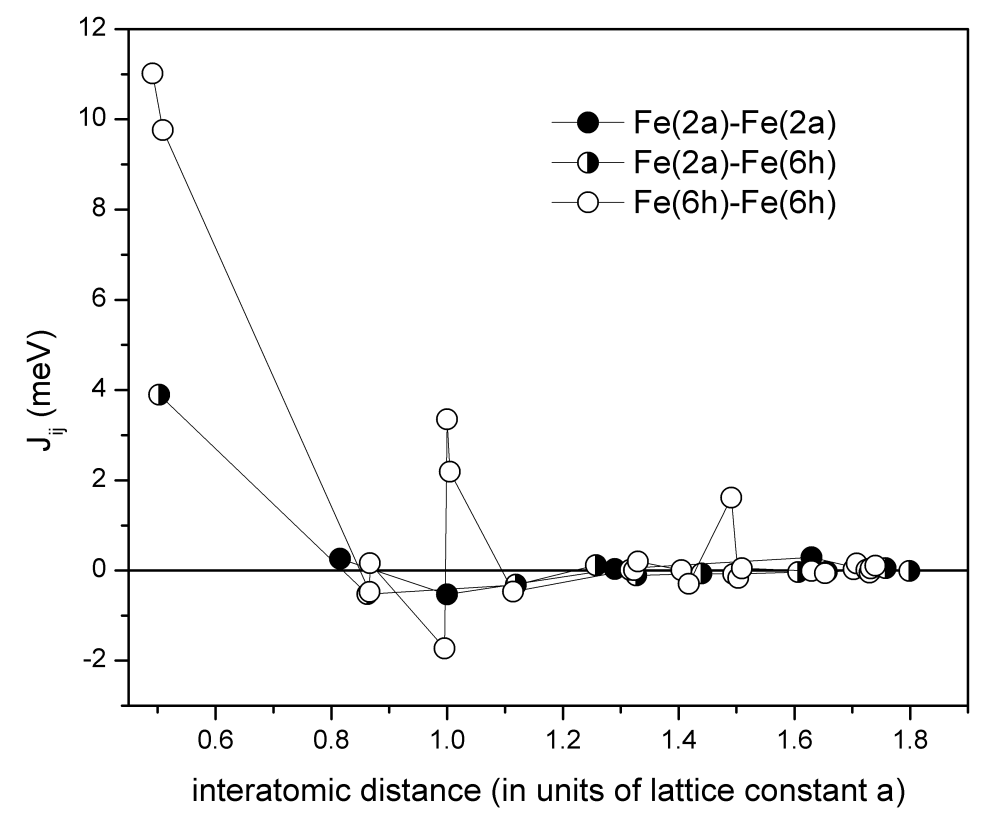

Figure 3. The inter-site exchange interactions in hexagonal $\mathrm{Fe}_{2} \mathrm{Hf}$ compound calculated in the disordered local moment (DLM) state. Open symbols are for the (2a)-(2a) Fe intra-sublattice interactions, closed symbols are for (6h)-(6h) intra-sublattice interactions, and semi-closed symbols denote the inter-sublattice (2a)-(6h) interactions. The calculations are presented for up to 50 nearest-neighbor shells. 


\section{2. $\mathrm{Fe}-\mathrm{Hf}-\mathrm{Sb}$ Alloy}

The MAE can be altered by alloying. Experimentally, Goll et al. [9] observed that off-stoichiometric $(\mathrm{Fe}, \mathrm{Sb})_{2+x} \mathrm{Hf}_{1-x}$ compounds have the uniaxial MAE of $\sim 1.5 \mathrm{MJ} \mathrm{m}^{-3}$. In order to examine the effect of $\mathrm{Sb}$ substitutional alloying as a possible tuning for positive MAE, we consider substitution of $\mathrm{Fe}$ and $\mathrm{Hf}$ atoms by $\mathrm{Sb}$ atoms in the $\mathrm{Fe}_{2} \mathrm{Hf}$ unit cell. To stay close to the experimental composition Fe60.0-Hf26.5-Sb13.5 [9], we substitute simultaneously one Fe and one $\mathrm{Hf}$ in the 12 atoms $\mathrm{Fe}_{2} \mathrm{Hf}$ unit cell. This yields Fe58-Hf25-Sb17 composition $\left(\mathrm{Fe}_{7} \mathrm{Hf}_{3} \mathrm{Sb}_{2}\right.$ unit cell) which we model considering 32 different ordered configurations of the Sb dopant.

We make use of the VASP code to evaluate the total energy. The total energies for different configurations of the $\mathrm{Sb}$ dopant are shown in Appendix A Table A1. We identify the four lowest energy ordered configurations where the Sb dopants substitute the pairs of [Fe (1)-Hf (4)] (see Table 1), and [Fe (1)-Hf (1)], [Fe (2)-Hf (2)], and [Fe (2)-Hf (3)]. All of these configurations in which Sb substitutes the Fe atoms in (2a) Wyckoff positions, are energetically equivalent. The alloy configuration where $\mathrm{Sb}$ dopants substitute the pair [Fe (1)-Hf (4)]) is shown in Figure 1B.

Additionally, we estimate stability of the lowest energy configurations calculating the enthalpy of formation $H$.

$$
H=E-\sum_{i} \mu_{i} x_{i}
$$

where $E$ is the DFT total energy of the alloy, $\mu_{i}$ is the chemical potential of element $i$, and $x_{i}$ is the quantity of element $i$ in the compound. The standard convention is to take the chemical potential of each species to be the DFT total energy of the elemental Fe, Hf, and Sb ground state. The enthalpy of formation is found $H=-0.724 \mathrm{eV} / \mathrm{f}$.u., which has somewhat smaller magnitude than the $H=-1.165 \mathrm{eV} /$ f.u. for pristine $\mathrm{Fe}_{2} \mathrm{Hf}$. Negative sign of $H$ supports the thermodynamic stability of Fe58-Hf25-Sb17 alloy.

Moreover, we estimate the enthalpy of formation for Fe58-Hf25-Sb17 alloy formed from pristine $\mathrm{Fe}_{2} \mathrm{Hf}$. In this case, the $\Delta H$ is calculated from the alloy total energy subtracting the total energy of $\mathrm{Fe}_{2} \mathrm{Hf}$, and taking into account excess of $\mathrm{Fe}$ and incorporation of two $\mathrm{Sb}$ atoms into the unit cell. This results in $\Delta H=0.15 \mathrm{eV} / \mathrm{f}$.u., and indicates the potential of $\mathrm{Sb}$ incorporation in already existing $\mathrm{Fe}_{2} \mathrm{Hf}$ at finite temperatures.

Next, we perform the FLAPW calculations for the alloy configuration shown in Figure 1B. The calculated magnetic moments inside MT-spheres for different Fe and Hf atoms in the unit cell together with the total magnetic moment per cell are shown in Table 1. It is seen that the magnetic moment is increased for the Fe atom in (2a) position, and is decreased for the (6h) Fe atoms. Additionally, magnitude of the moment on $\mathrm{Hf}$ atom is smaller than in the pristine $\mathrm{Fe}_{2} \mathrm{Hf}$. The $\mathrm{Sb}$ dopant yields almost no contribution to the magnetic moment, and the total moment per $\mathrm{Fe}_{7} \mathrm{Hf}_{3} \mathrm{Sb}_{2}$ unit cell is decreased by $3 \mu_{B}$.

Starting from the scalar-relativistic calculations, we perform the calculations with the SOC included, making use of a relativistic version of FLAPW [21]. In these calculations, the magnetization is fixed along [1100] and [0001] axes, as it was done for $\mathrm{Fe}_{2} \mathrm{Hf}$ described above, with the same set of the special k-points for the BZ-integration. The MAE of $0.88 \mathrm{meV}$ per unit cell $\left(0.82 \mathrm{MJ} \mathrm{m}^{-3}\right.$, see Table 2) is calculated. This significant positive MAE indicates the "out-of-plane" preferential direction of the magnetization, and is crucial for PM applications.

The calculations which are presented above were done assuming the experimental unit cell volume and internal positions of $\mathrm{Fe}_{2} \mathrm{Hf}$ [16]. The structure optimization can play important role, and we examined the effect of structural relaxation on the electronic structure, magnetism, and MAE of Fe-Hf-Sb alloy. In these calculations, the unit cell volume, c/a-ratio, and internal coordinates were optimized making use of the VASP method. Internal positions were relaxed until residual forces were less than $0.1 \mathrm{meV} / \AA$. The relaxed volume of $179 \AA^{3}$ and c/a-ratio of 1.64 for Fe58-Hf25-Sb17 alloy are bigger than the volume of $173 \AA^{3}$ and c/a-ratio of 1.63 for unrelaxed unit cell. The saturated magnetization of $10.93 \mu_{B}$ ( $0.57 \mathrm{MA} \mathrm{m}^{-1}$, see Table 2) slightly exceeds the unrelaxed value of 
$10.48 \mu_{B}\left(0.55 \mathrm{MA} \mathrm{m}^{-1}\right)$. This slight increase of the magnetization is consistent with an increase of equilibrium volume.

Importantly, the MAE is increased with relaxation from $0.88 \mathrm{meV}$ per unit cell $\left(0.82 \mathrm{MJ} \mathrm{m}^{-3}\right)$ to $1.37 \mathrm{meV}$ per unit cell $\left(1.27 \mathrm{MJ} \mathrm{m}^{-3}\right)$, as shown in Table 2 . The MAE calculations require special care to converge energy differences of often less than $0.1 \mathrm{meV}$ per unit cell from the total energy, which may be ten orders of magnitude larger. This is an involved endeavor and about 5200 special k-points in the full BZ were used in order to achieve the accuracy better than $0.05 \mathrm{meV}$ per unit cell in the MAE calculations.

Table 2. Magnetic moment $\left(M_{s a t}, \mathrm{MA} / \mathrm{m}\right)$, and the uniaxial magnetocrystalline anisotropy $\left(\operatorname{MAE}\left(K_{1}\right), \mathrm{MJ} / \mathrm{m}^{3}\right)$.

\begin{tabular}{ccc}
\hline Composition & $\boldsymbol{M}_{\text {sat }}$ & MAE \\
\hline unrelaxed Fe2Hf & 0.72 & -0.54 \\
unrelaxed Fe58-Hf25-Sb17 & 0.55 & +0.82 \\
relaxed Fe58-Hf25-Sb17 & 0.57 & +1.27 \\
\hline exp. Fe60-Hf26.5-Sb13.5 & $0.56-0.80$ & $1.4-1.5$ \\
\hline
\end{tabular}

Comparison between calculated relaxed Fe58-Hf25-Sb17 and experimental Fe60.0-Hf26.5-Sb13.5 [9] alloy is shown in Table 2. There is a fair agreement for the saturation magnetization $M_{s a t}$. The calculated value of $M_{s a t}$ is somewhat smaller since there is a deficiency of Fe and $\mathrm{Hf}$, and excess of $\mathrm{Sb}$ in the model supercell as compared with the experiment. Agreement between the uniaxial MAE is good. Significantly positive MAE and corresponding anisotropy field $H_{A} \approx 2 K_{1} / M_{\text {sat }}$ of $3.58 \mathrm{MA} / \mathrm{m}\left(\mu_{0} H_{A}=4.5 \mathrm{~T}\right)$, together with saturation magnetization of $0.72 \mathrm{~T}$ and reasonably high Curie temperature of $470 \mathrm{~K}$, allow us to suggest that Fe-Hf-Sb alloys with the optimal amount of $\mathrm{Sb}$ dopant can be a suitable candidates for a magnetically hard material.

\section{Discussion}

The MAE strength depends on the value of the SOC constant $\xi$ [22]. In Fe-Hf-Sb alloy, the SOC for $p$-states of $\mathrm{Sb}$, and $d$-states of Fe and Hf play a role for the MAE. The strongest $\xi=0.978 \mathrm{eV}$ is for the $5 p$-states of $\mathrm{Sb}$, while the $\xi$ values for $3 d$-states of Fe and $5 d$-states $\mathrm{Hf}$ are of $0.065 \mathrm{eV}$ and $0.188 \mathrm{eV}$ respectively. Bruno [22] has shown that the MAE is proportional to the anisotropy of the orbital moment $M_{L}$,

$$
\mathrm{MAE} \approx-\xi / 4\left(M_{L}\left\|[1100]-M_{L}\right\|[001]\right),
$$

when the exchange splitting $\xi<\Delta_{e x}$. For the Fe-Hf-Sb alloy, the exchange splitting $\Delta_{e x}=0.654 \mathrm{eV}$, and we can use this approach to estimate contributions to the MAE of Fe-Hf-Sb alloy from Fe and Hf atoms. Making use of the calculated $M_{L}$ values, we obtain the negative Fe-atoms MAE of $-1.14 \mathrm{meV}$ per unit cell, and the positive Hf-atoms MAE of $0.28 \mathrm{meV}$ per unit cell. The total contribution to the $\mathrm{MAE}=-0.88 \mathrm{meV}$ due to the SOC of Fe and $\mathrm{Hf} d$-states is semi-quantitatively consistent with the negative $\mathrm{MAE}=-0.57 \mathrm{meV}$ per unit cell for pristine $\mathrm{Fe}_{2} \mathrm{Hf}$.

Since the Sb-p-states' SOC is bigger than $\Delta_{e x}$, no perturbation theory is expected to be valid for their contribution to the MAE. At first, we show in Figure 4 the $p$-projected Sb-atoms' DOS for different directions of the magnetization. While they look alike, closer examination shows small $p$-DOS anisotropy of 0.02 states/eV at the Fermi level. This DOS anisotropy is usually related to the MAE [23].

In order to evaluate effect of the Sb-p-states SOC on the MAE, we performed the total energy calculations, where we set $\xi=0$ for the $p$-states of the $\mathrm{Sb}$ atoms; i.e., explicitly removed the $\mathrm{Sb} p$-state's contribution to the MAE. The calculated MAE $=0.13 \mathrm{meV}$ per unit cell $\left(0.12 \mathrm{MJ} / \mathrm{m}^{3}\right)$ is positive. It is substantially smaller than the full MAE of $1.37 \mathrm{meV}$ per unit cell $\left(1.27 \mathrm{MJ} / \mathrm{m}^{3}\right)$ shown in Table 2, 
where the Sb-p-states SOC is included. These calculations show explicitly and quantitatively that the $p$-states of Sb play a key role in the positive MAE of Fe58-Hf25-Sb17 alloy.
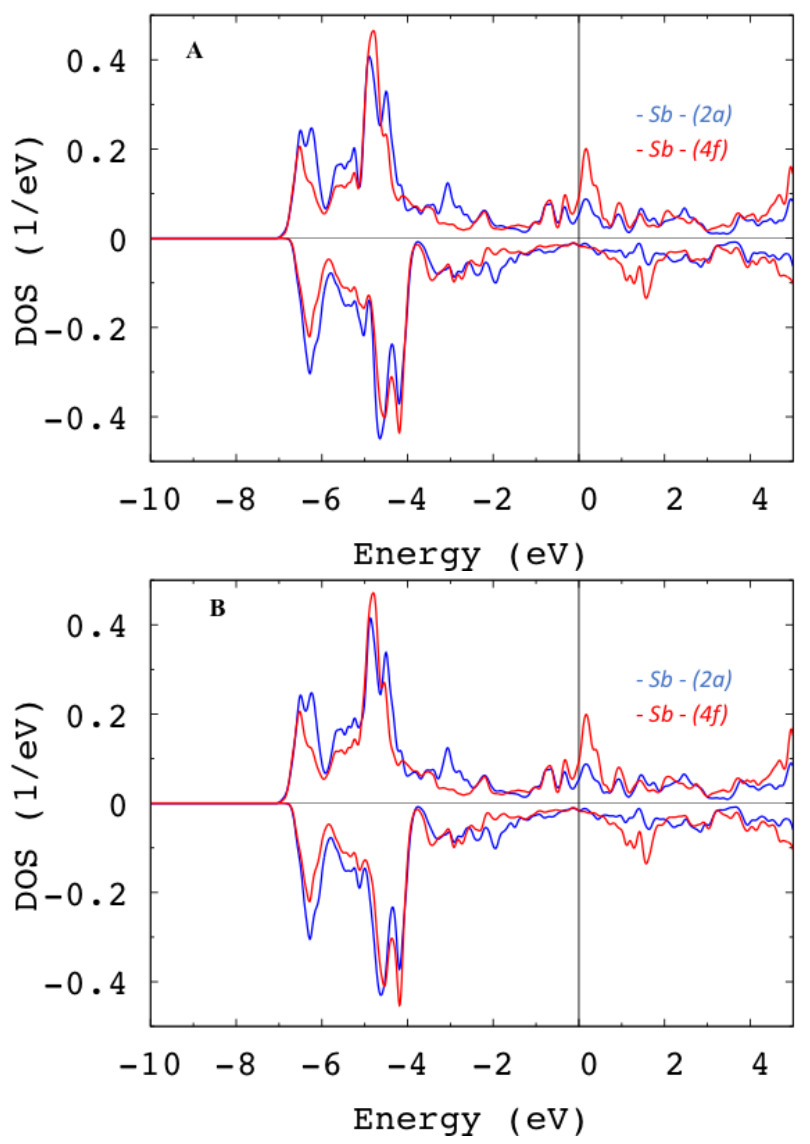

Figure 4. (A) Spin-projected $p$-DOS for $M||[0001]$; (B) spin-projected $p$-DOS for $M \| \mid[1100]$ for Sb atoms in (2a) and (4f) Wyckoff positions. The Fermi level $E_{F}$ is set to $E_{F}=0$.

\section{Conclusions}

To summarize, we have investigated the electronic structure and magnetic character of $\mathrm{Fe}_{2} \mathrm{Hf}$ hexagonal C14 phase. The negative MAE was found, and determined the "in-plane" preferential direction of the magnetization in the pristine material. The first-principles calculations demonstrate that $\mathrm{Sb}$ substitution changes the MAE from planar to uniaxial, in agreement with experimental observations. We emphasize the essential contribution of the structural relaxation to the MAE of Fe-Hf-Sb alloy. Our results suggest that the chemical substitution of the $p$-elements to the Fe-based hexagonal Laves phases can be a promising way for positive MAE control in the rare-earth free permanent magnets for technological applications.

Author Contributions: L.K. and M.T. performed the total energy VASP calculations for the electronic structure, crystal structure optimization, and enthalpy of formation; A.B.S. performed the total energy FLAPW calculations of the DOS, spin and orbital magnetic moments, and magnetic anisotropy energy; S.K. performed the KKR calculations of exchange coupling constants and Curie-temperature; D.L. performed the project administration, supervision, and analysis of the results. All authors contributed in writing the manuscript. All authors have read and agreed to the published version of the manuscript.

Funding: Financial support was provided by Operational Program Research, Development and Education financed by European Structural and Investment Funds and the Czech Ministry of Education, Youth and Sports (project number SOLID21-CZ.02.1.01/0.0/0.0/16_019/0000760), European Regional Development Fund in the IT4Innovations national supercomputing center-path to exascale project, project number CZ.02.1.01/0.0/0.0/16_013/0001791 within the Operational Program Research, Development and Education, mobility grant number 8J18AT004, and by the Czech Science Foundation grant number 18-06240S. D.L. was supported by the Czech Science Foundation grant number 17-23964S. 
Conflicts of Interest: The authors declare no conflict of interest.

\section{Appendix A}

The total energies relative to the lowest energy configuration for thirty two different configurations where the $\mathrm{Sb}$ dopants substitute the pairs of [Fe-Hf ] are shown in Table A1. We identify four different groups of the ordered configurations with the same energy. Inside of the each group, different configurations are connected by the symmetry operations.

Note that we made a comparison between the enthalpy of formation for Fe58-Hf25-Sb17 alloy $H_{\mathrm{Fe} 58-\mathrm{H} f 25-\mathrm{Sb} 17}=-0.724 \mathrm{eV} / \mathrm{f}$.u. (one Fe and one $\mathrm{Hf}$ atoms are substituted by $\mathrm{Sb}$ ), and two alloys where either Fe (Fe50-Hf33-Sb17) or Hf (Fe66-Hf17-Sb17) sites are replaced by Sb. We found $H_{\mathrm{Fe} 50-\mathrm{Hf33}-\mathrm{Sb} 17}=-0.179 \mathrm{eV} /$ f.u., and $H_{\mathrm{Fe} 66-\mathrm{Hf17}-\mathrm{Sb} 17}=-0.770 \mathrm{eV} /$ f.u. Thus, the configuration with two Hf sites substituted by Sb may be energetically preferable to the 1:1 Fe58-Hf25-Sb17 substitution. One has to take this comparison with some cautiousness since it is done for $\mathrm{T}=0 \mathrm{~K}$ and without phonon contribution.

Table A1. The total energy difference $\Delta E(\mathrm{eV} / \mathrm{f}$.u.) relative to the lowest energy configuration for 32 ordered configurations of $\mathrm{Fe}_{7} \mathrm{Hf}_{3} \mathrm{Sb}_{2}$.

\begin{tabular}{lcc}
\hline Group & Sb Substitution Sites & $\boldsymbol{\Delta} \boldsymbol{E}$ (eV/f.u) \\
\hline 1 & {$[\mathrm{Fe}(1)-\mathrm{Hf}(1)]$} & 0 \\
& {$[\mathrm{Fe}(2)-\mathrm{Hf}(2)]$} & 0 \\
& {$[\mathrm{Fe}(2)-\mathrm{Hf}(3)]$} & 0 \\
& {$[\mathrm{Fe}(1)-\mathrm{Hf}(4)]$} & 0 \\
\hline \multirow{2}{*}{} & {$[\mathrm{Fe}(3)-\mathrm{Hf}(1)]$} & 0.137 \\
& {$[\mathrm{Fe}(4)-\mathrm{Hf}(1)]$} & 0.137 \\
& {$[\mathrm{Fe}(7)-\mathrm{Hf}(1)]$} & 0.137 \\
& {$[\mathrm{Fe}(5)-\mathrm{Hf}(2)]$} & 0.137 \\
& {$[\mathrm{Fe}(6)-\mathrm{Hf}(2)]$} & 0.137 \\
& {$[\mathrm{Fe}(8)-\mathrm{Hf}(2)]$} & 0.137 \\
& {$[\mathrm{Fe}(3)-\mathrm{Hf}(3)]$} & 0.137 \\
& {$[\mathrm{Fe}(4)-\mathrm{Hf}(3)]$} & 0.137 \\
& {$[\mathrm{Fe}(7)-\mathrm{Hf}(3)]$} & 0.137 \\
& {$[\mathrm{Fe}(5)-\mathrm{Hf}(4)]$} & 0.137 \\
& {$[\mathrm{Fe}(6)-\mathrm{Hf}(4)]$} & 0.137 \\
& {$[\mathrm{Fe}(8)-\mathrm{Hf}(4)]$} & 0.137 \\
\hline \multirow{4}{*}{3} & {$[\mathrm{Fe}(5)-\mathrm{Hf}(1)]$} & 0.215 \\
& {$[\mathrm{Fe}(6)-\mathrm{Hf}(1)]$} & 0.215 \\
& {$[\mathrm{Fe}(8)-\mathrm{Hf}(1)]$} & 0.215 \\
& {$[\mathrm{Fe}(3)-\mathrm{Hf}(2)]$} & 0.215 \\
& {$[\mathrm{Fe}(4)-\mathrm{Hf}(2)]$} & 0.215 \\
& {$[\mathrm{Fe}(7)-\mathrm{Hf}(2)]$} & 0.215 \\
& {$[\mathrm{Fe}(5)-\mathrm{Hf}(3)]$} & 0.215 \\
& {$[\mathrm{Fe}(6)-\mathrm{Hf}(3)]$} & 0.215 \\
& {$[\mathrm{Fe}(8)-\mathrm{Hf}(3)]$} & 0.215 \\
& {$[\mathrm{Fe}(3)-\mathrm{Hf}(4)]$} & 0.215 \\
& {$[\mathrm{Fe}(4)-\mathrm{Hf}(4)]$} & 0.215 \\
& {$[\mathrm{Fe}(7)-\mathrm{Hf}(4)]$} & 0.215 \\
\hline & {$[\mathrm{Fe}(2)-\mathrm{Hf}(1)]$} & 0.258 \\
& {$[\mathrm{Fe}(1)-\mathrm{Hf}(2)]$} & 0.258 \\
& {$[\mathrm{Fe}(1)-\mathrm{Hf}(3)]$} & 0.258 \\
& {$[\mathrm{Fe}(2)-\mathrm{Hf}(4)]$} & 0.258 \\
\hline \multirow{4}{*}{4} & &
\end{tabular}




\section{References}

1. Skomski, R.; Coey, J.M.D. Permanent Magnetism; Institute of Physics: Bristol, UK, 1999.

2. Gutfleisch, O.; Willard, M.; Bruck, E.; Chen, C.; Sankar, S.; Liu, J. Magnetic Materials and Devices for the 21st Century: Stronger, Lighter, and More Energy Efficient. Adv. Mater. 2011, 23, 821-842. [CrossRef] [PubMed]

3. Skokov, K.P.; Gutfleisch, O. Heavy rare earth free, free rare earth and rare earth free magnets-Vision and reality. Scr. Mater. 2018, 154, 289. [CrossRef]

4. Herbst, J.M. R2Fe14B Materials-Intrinsic Properties and Technological Applications. Rev. Mod. Phys. 1991, 63, 819. [CrossRef]

5. Kumar, K. RETM5 and RE2TM17 Permanent Magnets Development. J. Appl. Phys. 1988, 63, R13. [CrossRef]

6. Zhang, H.; Dirba, I.; Helbig, T.; Alff, L.; Gutfleisch, O. Engineering perpendicular magnetic anisotropy in Fe via interstitial nitrogenation: N choose K. APL Mater. 2016, 4, 116104. [CrossRef]

7. Coey, J.M.D.; Sun, H. Improved magnetic properties by treatment of iron-based rare earth intermetallic compounds in ammonia. J. Magn. Magn. Mater. 1990, 87, L251. [CrossRef]

8. Vekilova, O.Y.; Fayyazi, B.; Skokov, K.P.; Gutfleisch, O.; Echevarria-Bonet, C.; Barandiaron, J.M.; Kovacs, A.; Fischbacher, J.; Schrefl, T.; Eriksson, O.; et al. Tuning the magnetocrystalline anisotropy of $\mathrm{Fe}_{3} \mathrm{Sn}$ by alloying. Phys. Rev. B 2019, 99, 024421. [CrossRef]

9. Goll, D.; Gross, T.; Loeffler, R.; Pflanz, U.; Vogel, T.; Kopp, A.; Grubesa, T.; Schneider, G. Hard Magnetic Off-Stoichiometric $(\mathrm{Fe}, \mathrm{Sb})_{2+x} \mathrm{Hf}_{1-x}$ Intermetallic Phase. Phys. Status Solidi RRL 2017, 11, 1700184. [CrossRef]

10. Engdahl, G. Handbook of Giant Magnetostrictive Materials; Engdahl, G., Ed.; Academic Press: San-Diego, CA, USA, 2000; Volume 264.

11. Levy, O.; Hard, G.L.W.; Curtarolo, S. Hafnium binary alloys from experiments and first principles. Acta Mater. 2010, 58, 2887. [CrossRef]

12. Villars, P.; Berndt, M.; Brandenburg, K.; Cenzual, K.; Daams, J.; Hulliger, F.; Massalski, T.; Okamoto, H.; Osaki, K.; Prince, A.; et al. The Pauling File. J. Alloys Compd. 2004, 367, 293. [CrossRef]

13. Kresse, G.; Joubert, D. From ultrasoft pseudopotentials to the projector augmented-wave method. Phys. Rev. B 1999, 59, 1758. [CrossRef]

14. Wimmer, E.; Krakauer, H.; Weinert, M.; Freeman, A.J. Full-potential self-consistent linearized-augmented-plane-wave method for calculating the electronic structure of molecules and surfaces: $\mathrm{O}_{2}$ molecule. Phys. Rev. B 1981, 24, 864. [CrossRef]

15. Ruban, A.V.; Skriver, H.L. Calculated surface segregation in transition metal alloys. Comput. Mater. Sci. 1999, 15, 119. [CrossRef]

16. Buschow, K.H.J.; van Engen, P.G.; Jongebreur, R. Magneto-optical properties of metallic ferromagnetic materials. J. Magn. Magn. Mater. 1983, 38, 1. [CrossRef]

17. Liechtenstein, A.I.; Katsnelson, M.I.; Antropov, V.P.; Gubanov, V.A. Local spin density functional approach to the theory of exchange interactions in ferromagnetic metals and alloys. J. Magn. Magn. Mater. 1987, 67, 65. [CrossRef]

18. Ruban, A.V.; Simak, S.I.; Shallcross, S.; Skriver, H.L. Local lattice relaxations in random metallic alloys: Effective tetrahedron model and supercell approach. Phys. Rev. B 2003, 67, 214302. [CrossRef]

19. Gyorffy, B.L.; Pindor, A.J.; Staunton, J.; Stocks, G.M.; Winter, H. A first-principles theory of ferromagnetic phase transitions in metals. J. Phys. F Met. Phys. 1985, 15, 1337. [CrossRef]

20. Khmelevskyi, S.; Ruban, A.V.; Mohn, P. Magnetic ordering and exchange interactions in structural modifications of $\mathrm{Mn}_{3} \mathrm{Ga}$ alloys: Interplay of frustration, atomic order, and off-stoichiometry. Phys. Rev. B 2016, 93, 184404. [CrossRef]

21. Shick, A.B.; Novikov, L.D.; Freeman, J.A. Relativistic spin-polarized theory of magnetoelastic coupling and magnetic anisotropy strain dependence: Application to Co/Cu (001). Phys. Rev. B 1997, 56, R14259. [CrossRef]

22. Bruno, P. Tight-binding approach to the orbital magnetic moment and magnetocrystalline anisotropy of transition-metal monolayers. Phys. Rev. B 1989, 39, 865. [CrossRef]

23. Shick, A.B.; Maca, F.; Masek, J.; Jungwirth, T. Prospect for room temperature tunneling anisotropic magnetoresistance effect: Density of states anisotropies in CoPt systems. Phys. Rev. B 2006, 73, 024418. [CrossRef]

(C) 2020 by the authors. Licensee MDPI, Basel, Switzerland. This article is an open access article distributed under the terms and conditions of the Creative Commons Attribution (CC BY) license (http://creativecommons.org/licenses/by/4.0/). 